\title{
ARTICLE OTX2 impedes self-renewal of porcine iPS cells through downregulation of NANOG expression
}

\author{
Ning Wang ${ }^{1}$, Yaxian Wang ${ }^{1}$, Youlong Xie and Huayan Wang
}

The transcription factor Otx2 acts as a negative switch in the regulation of transition from naive to primed pluripotency in mouse pluripotent stem cells. However, the molecular features and function of porcine OTX2 have not been well elucidated in porcineinduced pluripotent stem cells (piPSCs). By studying high-throughput transcriptome sequencing and interfering endogenous OTX2 expression, we demonstrate that OTX2 is able to downgrade the self-renewal of piPSCs. OTX2 is highly expressed in porcine brain, reproductive tissues, and preimplantation embryos, but is undetectable in fibroblasts and most somatic tissues. However, the known piPSC lines reported previously produced different levels of OTX2 depending on the induction procedures and culture conditions. Overexpression of porcine OTX2 can reduce the percentage of alkaline phosphatase-positive colonies and downregulate NANOG and OCT4 expression. In contrast, knockdown of OTX2 can significantly increase endogenous expressions of NANOG, OCT4, and ESRRB, and stabilize the pluripotent state of piPSCs. On the other hand, NANOG can directly bind to the OTX2 promoter as shown in ChIP-seq data and repress OTX2 promoter activity in a dose-dependent manner. These observations indicate that OTX2 and NANOG can form a negative feedback circuitry to regulate the pluripotency of porcine iPS cells.

Cell Death Discovery (2016) 2, 16090; doi:10.1038/cddiscovery.2016.90; published online 5 December 2016

\section{INTRODUCTION}

Pluripotent stem cells have two pluripotency states, naive and primed, depending on the origin of cells and the culture conditions. ${ }^{1}$ In mouse, embryonic stem cells (ESCs) derived from the inner cell mass in the early stage of the blastocyst retain naive pluripotency, whereas epiblast stem cells derived from the epiblast in the late stage of the blastocyst retain primed pluripotency. ${ }^{1-3}$ Clear distinctions between the two pluripotent states have been defined on the basis of cell morphology, cytokine supplementation, signaling pathways, formation of chimera and germline transmission in vivo, and transcriptomics. Naive pluripotent stem cells show compact and dome-like colonies, depend on leukemia inhibitory factor (LIF), and are able to generate germline transmitting chimeras. ${ }^{1,2,4,5}$ In contrast, primed pluripotent stem cells form flattened colonies, depend on basic fibroblast growth factor (bFGF), and show very limited capacity to generate chimeric offspring. ${ }^{2,6,7}$ Furthermore, a set of transcription factors (TF), such as Nanog, Oct4, Esrrb, and Otx2, has been shown to regulate the transaction between naive and primed pluripotent states. ${ }^{1,8}$

The TF Otx2, which plays an essential role in the regulation of brain and sense organ development and neuronal differentiation, ${ }^{9-11}$ is a crucial regulator in the transition of murine naive ESCs into primed epiblast stem cells. ${ }^{12}$ Increasing the activity of Otx2 causes a significant enrichment of FGF protein expression and reduces the generation of chimeric embryos. Conversely, absence of Otx2 leads to an increase of LIF/STAT3 signaling activity. ${ }^{12}$ Genome-wide mapping of enhancer activity and protein-DNA interaction profiles show that Otx2, as a cell-statespecific regulator, can interact with Oct4 and combine with primed-dominant enhancers to drive the reorganization of enhancer usages during differentiation. ${ }^{13}$ Therefore, Otx2/Oct4bound enhancers are important for maintaining cellular identity and leading pluripotent stem cells to exit from naive state pluripotency. ${ }^{14}$ In addition, knockdown of Otx2 leads the primed state cells to flip to naive ESCs by increasing the expression of Esrrb that is directly regulated by Nanog, and Esrrb can form a binding partner with Oct4 to bind to naive-dominant enhancers. ${ }^{14,15}$ Thus, Otx2 may have a mutually exclusive effect on Nanog, which is well studied as a critical factor on blocking the differentiation of pluripotent stem cells ${ }^{16,17}$ and maintaining the pluripotent state of stem cells. ${ }^{12}$

Porcine-induced pluripotent stem cells (piPSCs) have been reported by several laboratories worldwide. ${ }^{18-24}$ Some piPSC lines showed the primed state with bFGF-dependence and mouse epiblast stem cells-like morphology ${ }^{18-21}$ other piPSC lines showed the naive-like state with LIF-dependence and mouse ESC-like morphology. ${ }^{23}$ Besides, piPSCs cultured with both LIF and bFGF represented the metastable state. ${ }^{24}$ So far, porcine ESCs and naive state piPSCs are difficult to generate. The underlying problems are improper culture conditions used to generate the piPSCs and unclear cell-state-specific regulatory circuitries. Thus, naive piPSC generation will benefit from an understanding of the genetic and epigenetic mechanisms that control the self-renewal and differentiation of piPSCs.

In our previous studies, mRNA expression profiles showed clear differences in the expression status of TFs in LIF-dependent, ${ }^{23}$ bFGF-dependent, ${ }^{18}$ and LIF/bFGF-dependent piPSCs. ${ }^{24}$ Interestingly, OTX2 expression in LIF-dependent piPSCs was distinguished from that in bFGF-dependent piPSCs, suggesting that OTX2 might act as a molecular marker to classify the different pluripotent states of pig iPSCs. In this study, we explored whether OTX2 was 
functionally relevant to the pluripotency of piPSCs. Also, we dissected the relationship between OTX2 and NANOG in such regulation.

\section{RESULTS}

Porcine OTX2 expression pattern

In porcine tissues, including testis, ovary, and brain, OTX2 was highly expressed (Figure 1a). The qRT-PCR analysis further confirmed that OTX2 expression in testis, ovary, and brain was 8-17-fold higher than that in other tissues (Figure 1b). Since OTX2 was reported to be relevant to embryo development, ${ }^{25,26}$ we then investigated the OTX2 expression in porcine embryos. Results of reverse transcription polymerase chain reaction (RT-PCR) and quantitative RT-PCR (qRT-PCR) showed high OTX2 expression in oocytes, indicating that OTX2 is a maternal factor; OTX2 expression increased in the preimplantation embryos during the zygotic transition starting from the eight-cell stage (Figures 1c and d). In cell-based experiments, OTX2 was expressed in porcine LIF/bFGFdependent iPS cells-piPS-g ${ }^{24}$ and PS23 ${ }^{22}$ cells, but not in somatic porcine embryonic fibroblasts (PEF; Figure 1e). This observation allowed us to use these cell lines to explore the function of OTX2. RNA-seq data (E-MTAB-2634) showed that OTX2 was highly expressed in bFGF-dependent iPS cells (piPS-w), ${ }^{18}$ but OTX2 expression was rather low in LIF-dependent iPS cells (piPS-j). ${ }^{23}$ In LIF/bFGF-dependent iPS cells (piPS-g), OTX2 expression fell somewhere between piPS-w and piPS-j (Figure 1f). Expression patterns of endogenous OTX2 in piPSCs were confirmed by qRT-PCR (Figure 1g).

\section{Characterization of porcine OTX2}

Phylogenetic analysis showed that pig OTX2 was evolutionarily related to cattle OTX2, whereas human, baboon, marmoset, rat, and mouse shared the most Otx2 sequence identity (Figure 2a). Porcine OTX2 CDS was amplified from porcine brain tissue in which OTX2 is an essential factor for brain development, ${ }^{12}$ confirmed by DNA sequencing, and submitted to GenBank (Accession No. KP779653). BLAST research found that the porcine OTX2 gene was located on the complementary strand of chromosome 1 and contained three exons (Figure 2b). Alignment of the cloned OTX2 CDS with the pig genome sequence showed 99\% identity (Supplementary Figure S1).

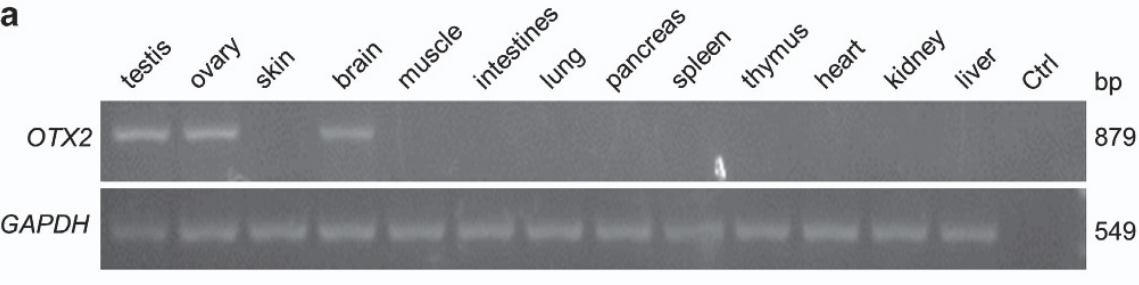

b

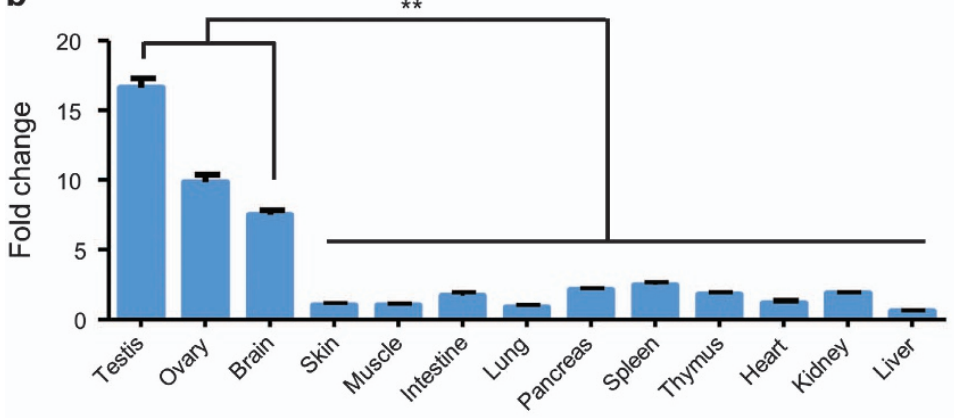

C
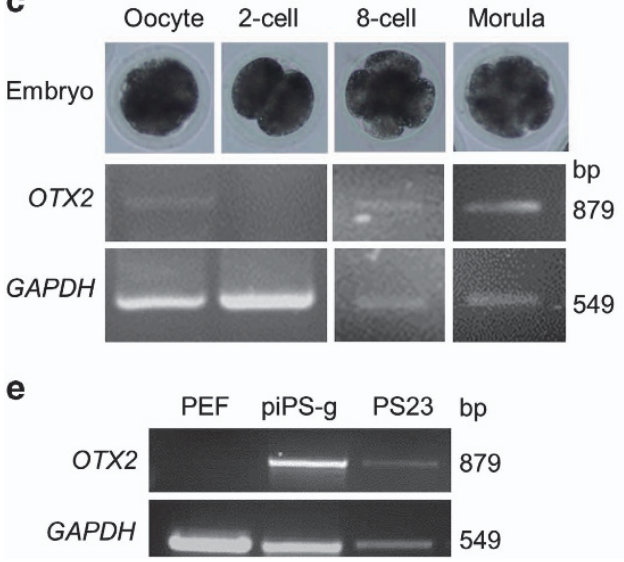

f
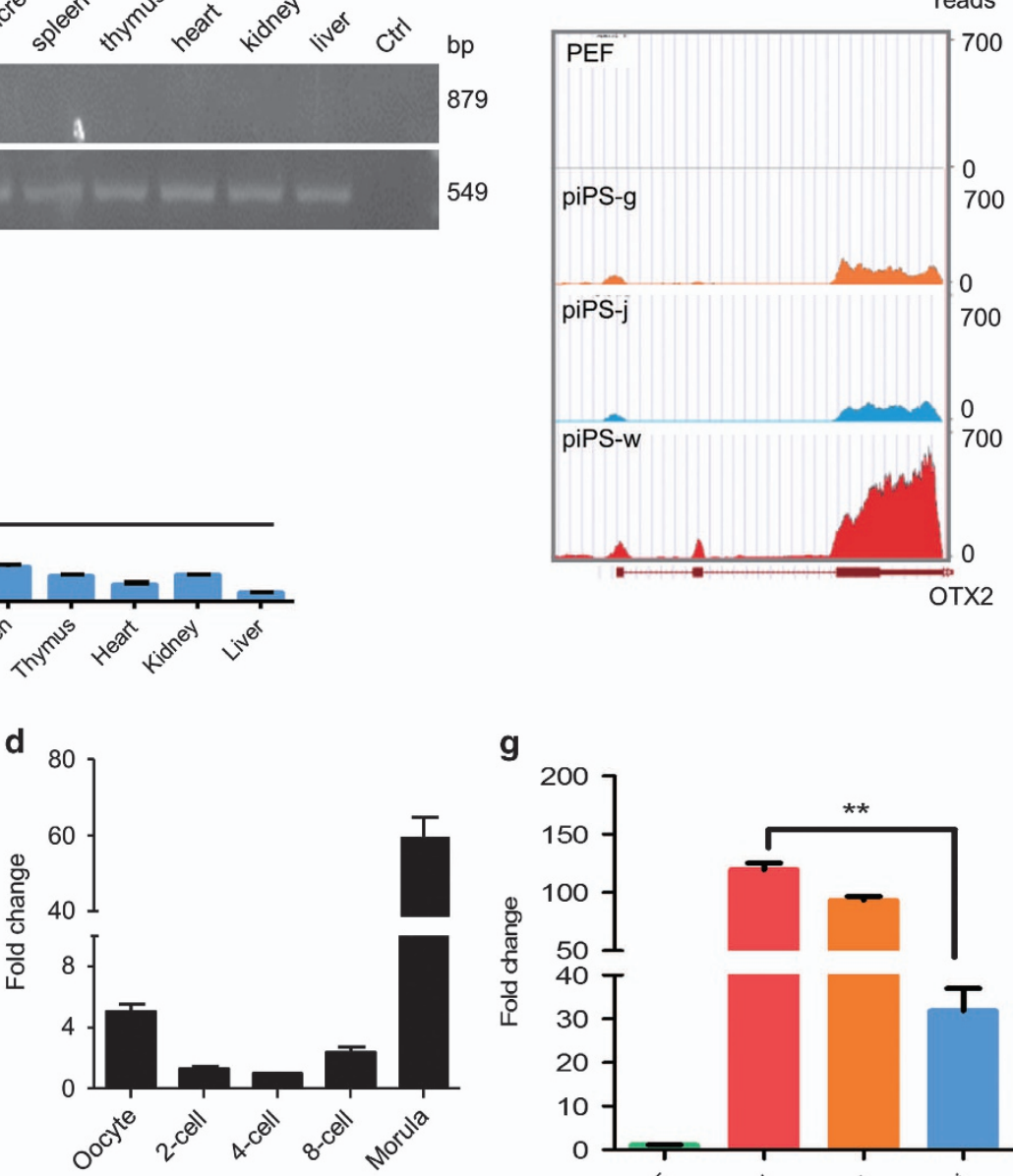

g

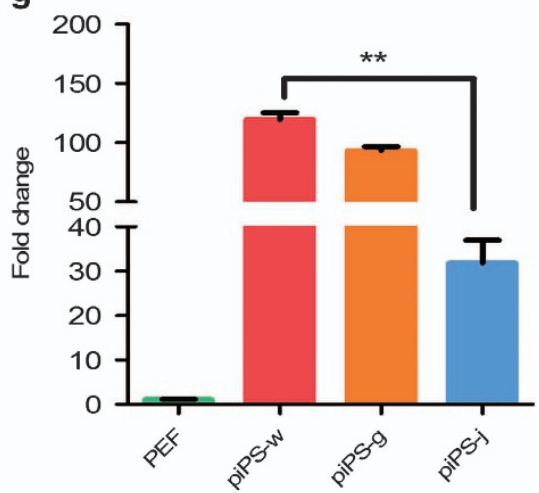

Figure 1. Expression pattern of OTX2 in porcine tissues and cell lines. OTX2 expressions in porcine tissues and cell lines were determined by RT-PCR and transcriptome sequencing. (a and b) OTX2 expression in porcine tissues. Ctrl, negative control. (c and d) OTX2 expression in porcine oocytes and parthenogenetic preimplantation embryos. (e) OTX2 expression in somatic (PEF) and pluripotent (piPS-g and PS23) cells. (f) Transcriptome reads of OTX2 from PEF and porcine iPS cell lines. (g) qRT-PCR analysis of OTX2 expression in PEFs, piPS-w, piPS-g, and piPS-j cells. ${ }^{* *} P<0.01, n=3$. 
a

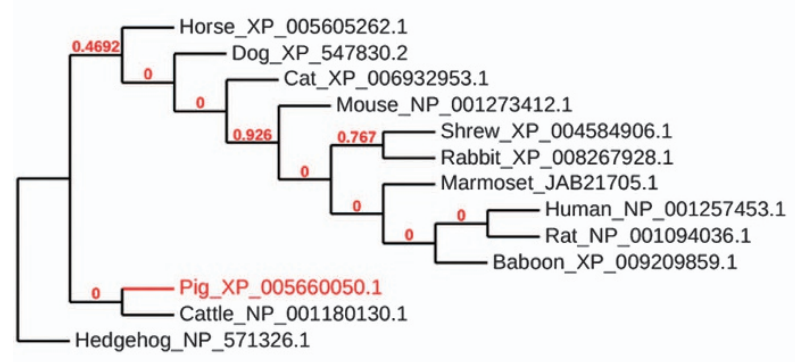

b

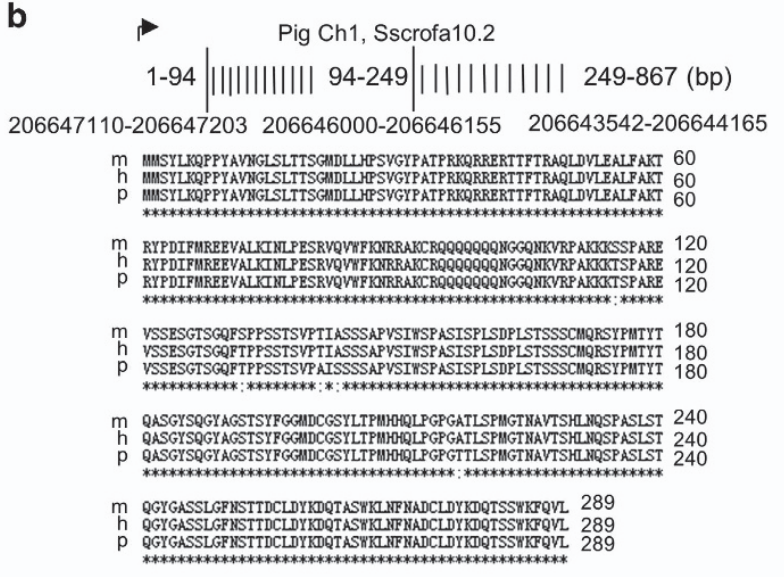

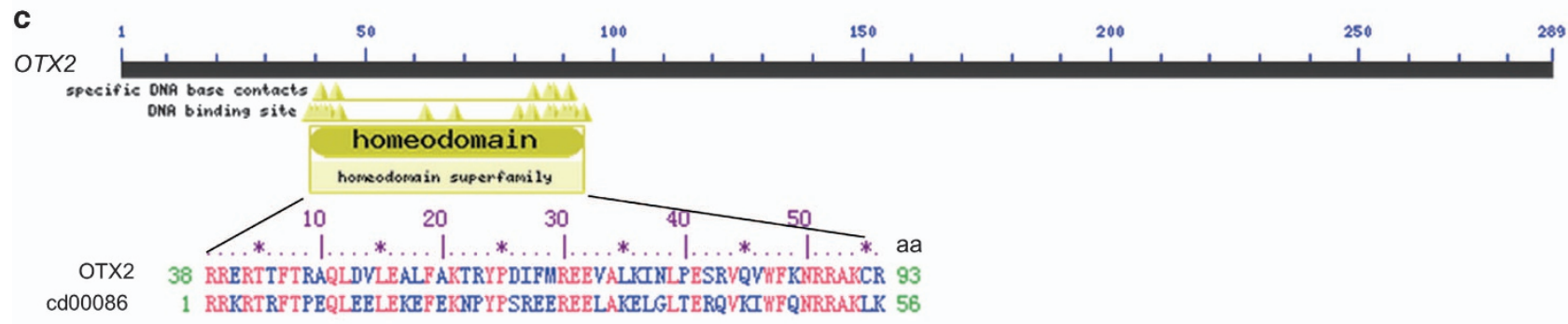
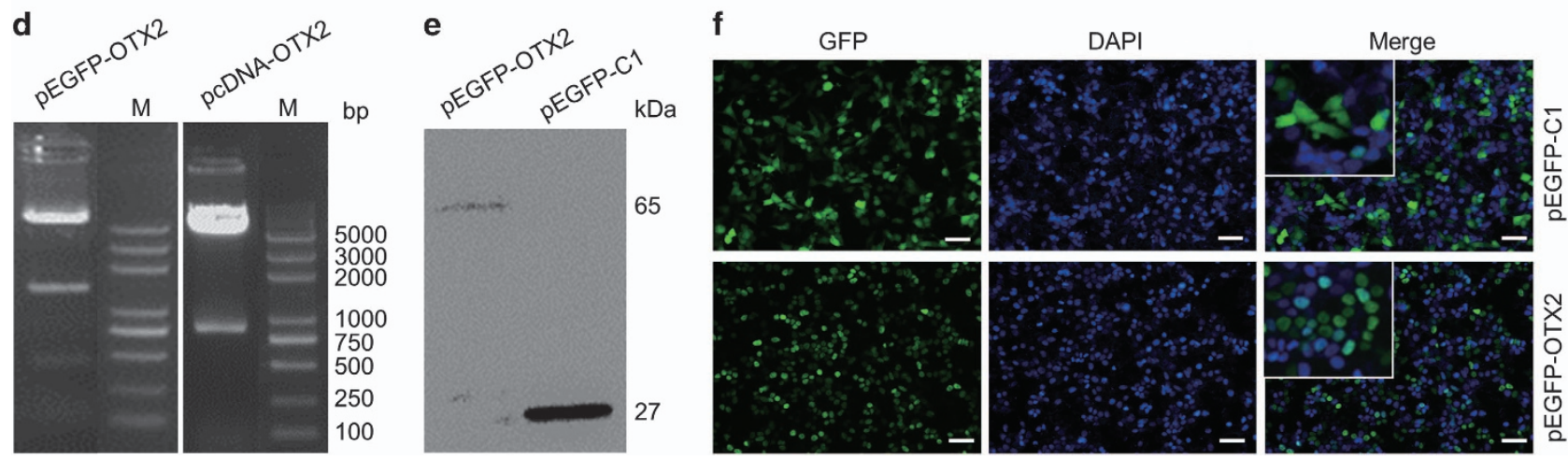

Figure 2. Characterization of porcine OTX2. (a) Phylogenetic tree of OTX2 protein calculated by online phylogenetics software. (b) Alignment of OTX2 protein sequences among animal species mouse (m), human (h), and pig (p). (c) Conserved domain of porcine OTX2 that retains a homeodomain sequence with 56 amino acids. Cd00086 denotes the accession number of the homeodomain sequence in the Conserved Domain Database. (d) Restriction enzyme digestions of pEGFP-OTX2 and pcDNA-OTX2. M, D2000 plus DNA ladder. (e) Western blotting analysis of GFP protein (27 kDa) and GFP-OTX2 fusion protein (65 kDa) in HEK-293 T cells. (f) GFP fluorescent assay. After a 36-h transfection, GFP- and GFP-OTX2-positive cells were observed by fluorescence microscopy. The inset is the enlarged image. Nuclei were stained with DAPI (blue). Scale bar, $50 \mu \mathrm{m}$.

The $2 \mathrm{~kb}$ OTX2 promoter fragment and an $892 \mathrm{bp}$ fragment of $3^{\prime}$ untranslated region ( 3 ' UTR) were also cloned from porcine brain tissue, and confirmed by DNA sequencing (Supplementary Figure S2). The porcine OTX2 promoter sequence was submitted to GenBank (Accession No. KR135411).

The protein sequence of OTX2 has 288 amino acids (aa) and exhibits high homology with human and mouse Otx2 (Figure 2b). On the basis of the Conserved Domain Database, 27,28 OTX2 belongs to the OTX TF super family (Accession No. pfam03529). OTX2 has a homeodomain sequence with 56 amino acids (Accession No. cd00086; Figure 2c), which is predicted to be involved in DNA binding and homodimer or heterodimer formation in a sequence-specific manner.

To investigate the function of OTX2 in eukaryotic cells, we constructed the expression vectors pEGFP-OTX2 and pcDNA-OTX2, which were confirmed by enzyme digestion (Figure 2d). The pEGFPOTX2 and a control pEGFP-C1 were transfected into HEK-293 T cells for $36 \mathrm{~h}$, and the presence of EGFP-OTX2 fusion protein was confirmed by western blotting (Figure 2e). In a cell-based assay, EGFP-positive and EGFP-OTX2-positive cells were observed with fluorescence microscopy (Figure 2f). EGFP-OTX2 fusion protein was translocated in nuclei, whereas EGFP protein was displayed in whole cells (Figure 2f, inset). Thus, the cloned OTX2 CDS can be transcribed into functional protein and be used for further research.

OTX2 influences morphology and expression of pluripotent genes in piPSCs

To investigate the regulatory function of OTX2 in piPSCs, the piPS PS23 cells were transfected by pcDNA-OTX2. Western blotting confirmed that cells transfected with pcDNA-OTX2 expressed high-level OTX2 protein (Figure 3a). Alkaline phosphatase (AP) staining showed that OTX $2^{+}$piPSC colonies were partially reduced the AP staining compared with the control group, which displayed 
a
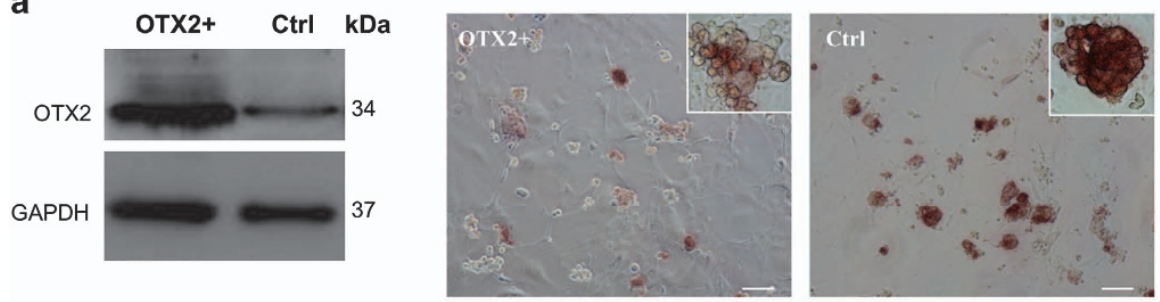

b

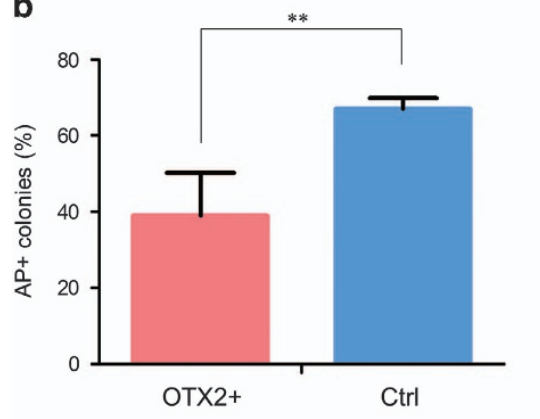

C

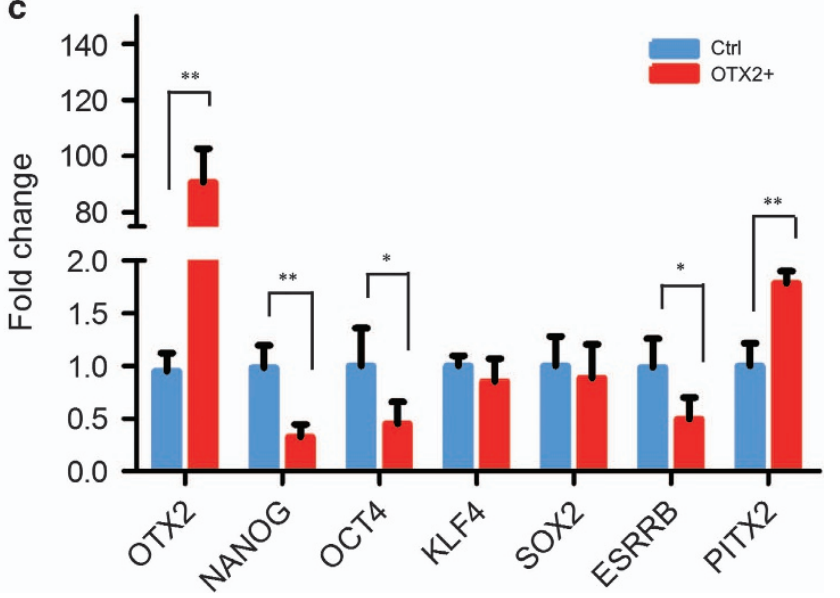

Figure 3. Overexpression of OTX2 in piPSCs. (a) Western blotting analysis of OTX2 protein expression and AP staining in OTX2 transfected PS23 cells. Scale bar, $50 \mu \mathrm{m}$. (b) Quantitative analysis of AP-positive colonies. (c) Quantitative RT-PCR analysis of pluripotent gene expressions in PS23 cells. OTX2+, piPS cells were transfected with pCDNA-OTX2; Ctrl, piPS cells without the pcDNA-OTX2 transfection. ${ }^{*} P<0.05,{ }^{* *} P<0.01, n=3$.

a more compact morphology with uniform AP staining (Figure 3a). The proportion of $\mathrm{AP}^{+}$colonies in $\mathrm{OTX} 2^{+}$piPSCs was significantly lower than that in the control group (Figure 3b), indicating that the pluripotent state of piPSCs was disturbed by ectopic OTX2 overexpression. To further uncover changes in self-renewal, we then examined endogenous pluripotent gene expression in OTX2 ${ }^{+}$ piPSCs. The level of OTX2 in OTX2 $2^{+}$piPSCs was significantly increased comparing with the endogenous level of OTX2 in piPSCs (Figure 3c). However, the expression level of NANOG was reduced more than $70 \%$ and the expression levels of OCT4 and ESRRB were reduced to $50 \%$ in $\mathrm{OTX}^{+}$piPSCs. Conversely, the expression of PITX2, a differentiation-related gene, was significantly increased (Figure 3c). Interference in the expression of other core pluripotent genes, such as SOX2 and KLF4, by OTX2 overexpression was observed, but was not statistically significant (Figure 3c). This observation proved the concept similar to the recently reported results that overexpression of OTX2 significantly downregulated expression of pluripotent genes NANOG, OCT4, SOX2, and so on. and decreased the self-renewal, survival and cell migration in human ES cells. ${ }^{29}$

The RNA interfering assay showed that expression of OTX2 in both DNA level and protein level was significantly knocked down by all three siRNAs, in which siR-543 and siR-1115 caused $95 \%$ reduction in OTX2 expression (Figure 4a). Besides, the AP staining showed that piPSCs treated with siRNAs exhibited much more compact clones compared with the control group (Figure 4b). Moreover, statistics data showed that the percentage of fine AP-stained colonies in siR-543 treatment was significantly higher than that in a control group (Figure 4c). Additionally, we found that the expressions of endogenous NANOG, OCT4, and ESRRB significantly increased in piPSCs, and PITX2, a factor that regulates cell differentiation, was remarkably downregulated in the presence of reduced OTX2 expression (Figure 4d). Interestingly, SOX2 expression was reduced significantly when OTX2 was knocked down, suggesting that further investigation of SOX2 function might reveal its role in regulating the pluripotent state in piPSCs. These observations indicated that OTX2 knockdown could stabilize the self-renewal and reduce the differentiation potential of piPSCs.

\section{OTX2 downregulates NANOG expression}

It has been reported in mouse embryos that loss of Otx2 could severely affect Nanog expression. ${ }^{12}$ RNA-seq data generated from different stages of porcine embryos showed that endogenous NANOG was highly expressed in eight-cell embryos, whereas OTX2 was highly expressed at the morula stage (Figure 5a). ${ }^{30}$ Transcriptome analysis, confirmed with qRT-PCR, showed that OTX2 expression increased significantly in the morula stage, and NANOG expression at the eight-cell stage was twofold higher than that at four-cell and morula stages (Figure $5 \mathrm{~b}$ ). In previous experiments (Figure 3c), we noticed that endogenous NANOG expression was significantly downregulated in $\mathrm{OTX2}^{+}$piPSCs, suggesting that OTX2 might play a role in negatively regulating NANOG expression. To determine the effect of OTX2 on NANOG expression, the pcDNA-OTX2 and reporter vector pGL-NANOG were transiently cotransfected into HEK-293 T cells in which both OTX2 and NANOG were not expressed (Figure 5c). Luciferase assays showed that the activity of the NANOG promoter could be remarkably downregulated by OTX2 in both time-dependent and dose-dependent manners (Figure $5 \mathrm{~d}$ ). To further investigate activation of the NANOG promoter, expression vectors carrying OTX2, OCT4, and SOX2 were cotransfected with reporter pGLNANOG into HEK-293 T cells, respectively. Results showed that addition of OCT4 and SOX2 significantly enhanced NANOG promoter activity, similar to a previous study. ${ }^{31}$ However, addition of OTX2 alone significantly reduced NANOG promoter activity (Figure 5e). To investigate the synergistic effect of combining OTX2 with OCT4/SOX2, we applied OTX2 with OCT4 and SOX2. 
a
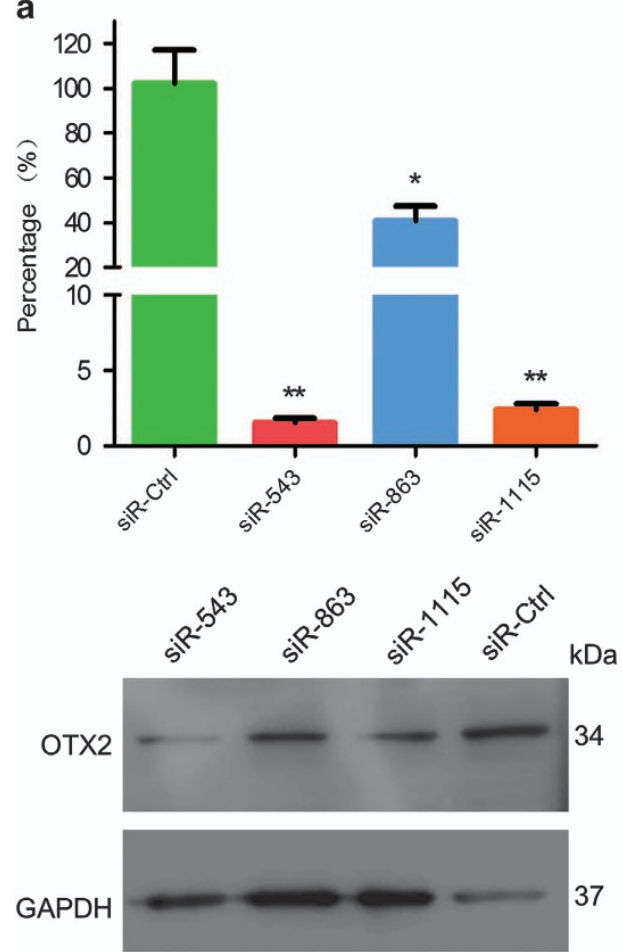

b
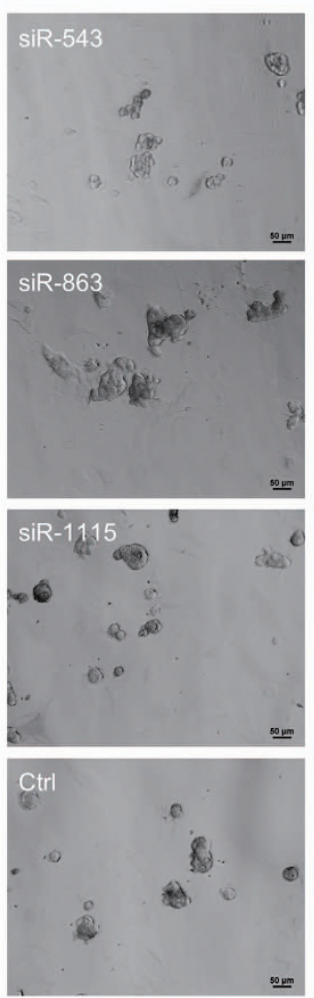

Phase
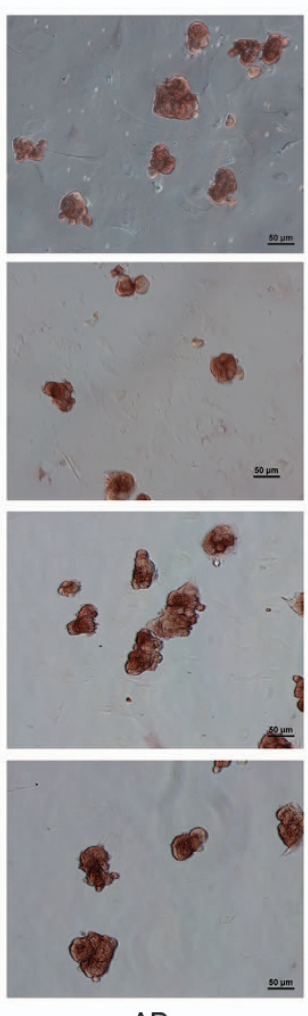

C

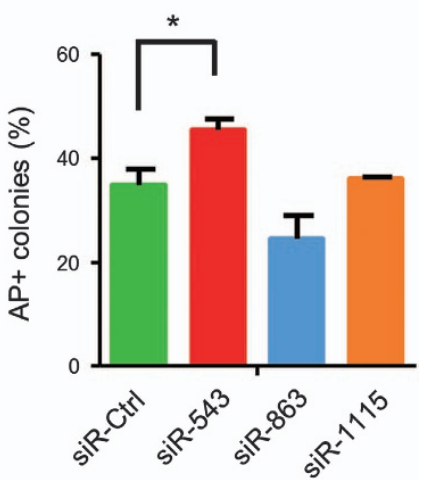

d

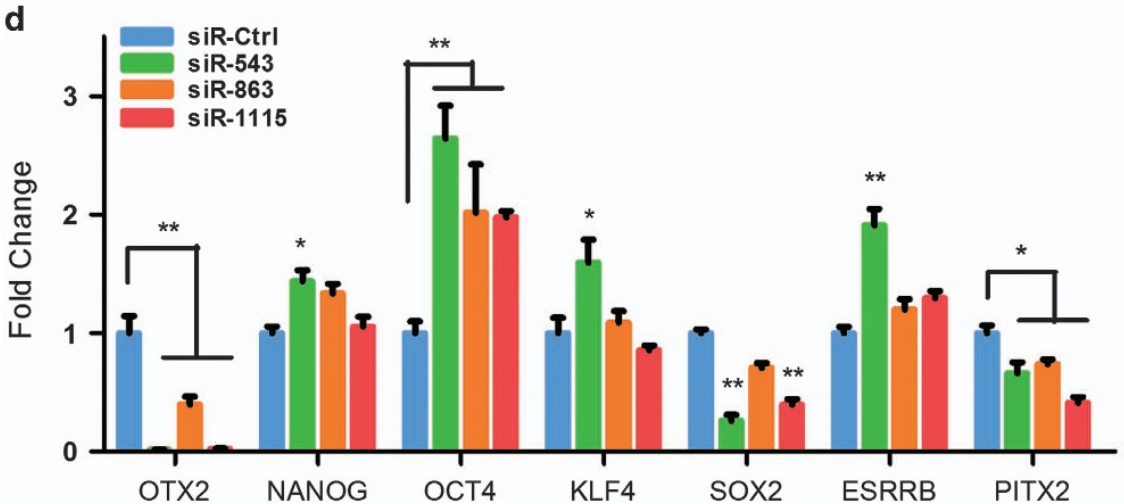

Figure 4. Knockdown of OTX2 expression in piPSCs. (a) Quantitative RT-PCR and western blotting analyses of OTX2 expression in PS23 cells that were treated with siRNAs for 36 h. (b) AP staining of PS23 cells with siRNAs' treatment. Scale bar, $50 \mu \mathrm{m}$. (c) Quantification of AP-positive clones. (d) qRT-PCR analysis of pluripotent gene expression in PS23 cells with siRNAs' treatment. siR-Ctrl, PS23 cells were transfected with control siRNA. ${ }^{*} P<0.05,{ }^{* *} P<0.01, n=3$.

NANOG promoter activity increased slightly compared with the treatment lacking addition of OTX2, but the increase was not statistically significant (Figure 5 e). This result suggests that OTX2 and OCT4/SOX2 do not have a synergistic effect on the activity of porcine NANOG; however, the exclusive application of OTX2 does impede NANOG promoter activation.

To further investigate OTX2 regulation of NANOG expression, we constructed a series of reporter constructs of porcine NANOG, which retained the truncated promoter sequence reported previously. ${ }^{31}$ Within the promoter sequence, seven putative OTX2 binding sites and multiple putative OCT4 and SOX2 binding sites were found on the basis of the online JASPAR software (Figure $5 f$, left). Double enzyme digestions confirmed the accuracy of these recombinant vectors (Figure $5 \mathrm{f}$, right). NANOG reporter pGL-NANOG and three truncated constructs pGL-N1, pGL-N2, and pGL-N3 were transiently cotransfected with pcDNA-OTX2 into HEK-293 T cells, respectively. The promoter activity was significantly repressed in cells transfected with pGL-N3 and pGL-NANOG, but not in cells with pGL-N1 and pGL-N2 (Figure $5 \mathrm{~g}$ ). These results indicated that OTX2 could block activation of the NANOG promoter and its binding sites were probably located in the distal region of the NANOG promoter. In the future study, the gel shift assay and ChIP-seq experiment might reveal whether OTX2 binds directly or indirectly to the NANOG promoter.

NANOG directly regulates OTX2 expression

ChIP-seq datasets from mouse, human, and pig showed that Nanog could directly bind to the mouse and human Otx2 promoter region. However, pig ChIP-seq profiles exhibited numerous noisy due to the quality of the anti-NANOG antibody 
a

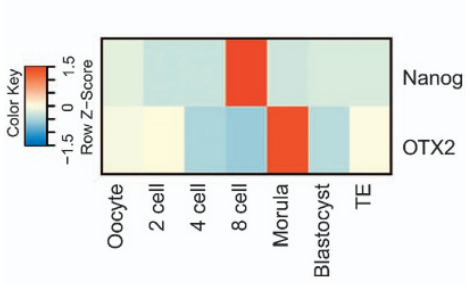

b

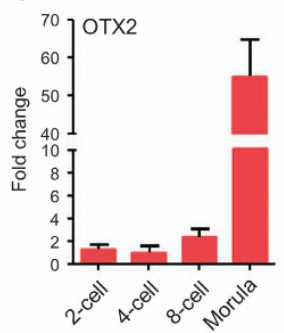

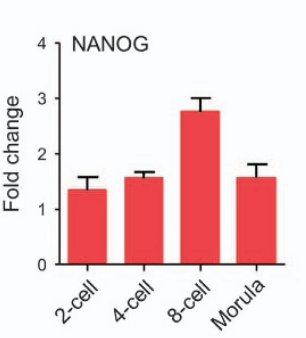

c

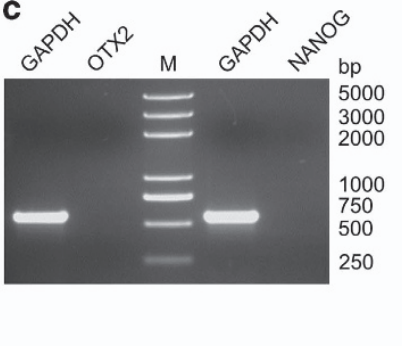

d
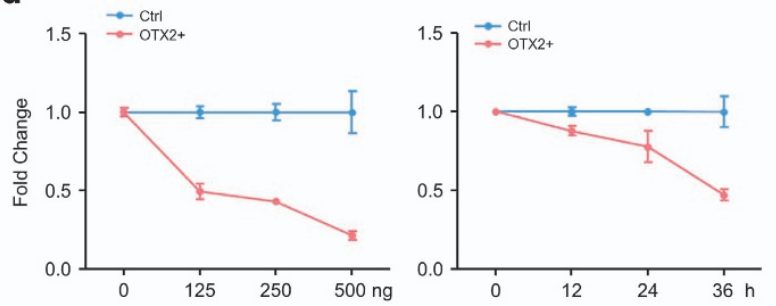

e

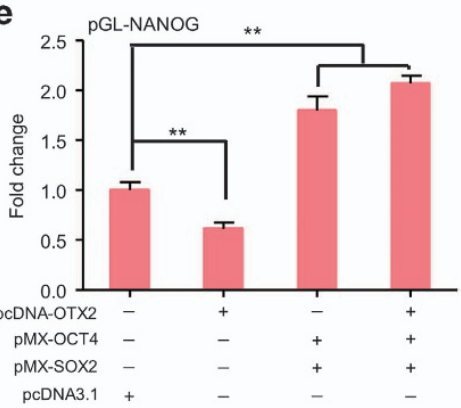

f

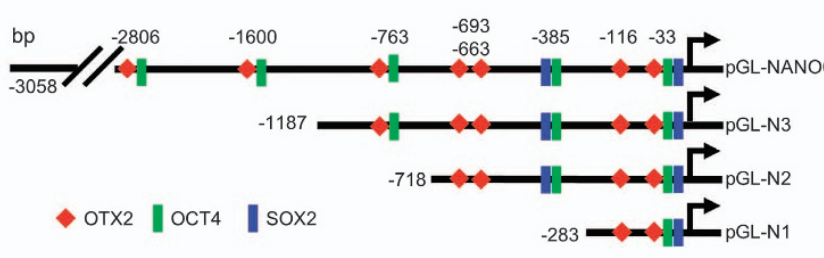

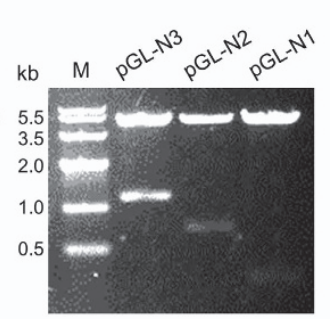

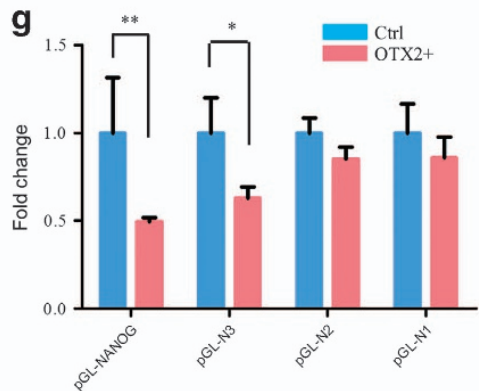

Figure 5. OTX2 downregulates NANOG expression. (a) Expression profile of OTX2 and NANOG in pig oocyte and parthenogenetic preimplantation embryos. TE, Trophectoderm. (b) qRT-PCR analysis of OTX2 and NANOG in two-cell, four-cell, eight-cell, and morula embryos. (c) RT-PCR analysis of OTX2 and NANOG in HEK-293 T cells. M, D2000 plus DNA ladder. (d) pcDNA-OTX2 and pGL-NANOG were cotransfected into HEK-293 T cells for $36 \mathrm{~h}$. Dose-dependent assay (left) was performed with various concentrations of pcDNA-OTX2. Time-dependent assay (right) was performed with $250 \mathrm{ng}$ of PCDNA-OTX2 for various time points. Ctrl, cells were only transfected with reporter pGL-NANOG. (e) Luciferase assay of NANOG promoter activation. The expression vectors carrying with OTX2, OCT4, and SOX2 were cotransfected with pGLNANOG into HEK-293 T cells for $36 \mathrm{~h}$, respectively. The pcDNA 3.1 vector was used as a control. (f) Truncated constructs of the NANOG promoter with predicted DNA binding sites were confirmed by Hindlll and Xho I double digestion. M, DNA Marker IV. (g) HEK-293 T cells were cotransfected with pCDNA-OTX2 and truncated NANOG promoter vectors for $36 \mathrm{~h}$. NANOG promoter activity was determined by the luciferase assay. Ctrl, cells were only transfected with reporter pGL-NANOG. ${ }^{*} P<0.05,{ }^{* *} P<0.01, n=3$.

that lacks pig specificity (Figure 6a). ${ }^{32}$ To investigate whether NANOG could regulate porcine OTX2 expression, we cloned the $2 \mathrm{~kb}$ porcine OTX2 promoter and constructed OTX2 reporter vectors POTX2-GFP and pGL-OTX2. The POTX2-GFP and pEGFP-C1 were cotransfected into PEFs, a porcine epithelial cell line (PK15), and a porcine iPS cell line (PS23). Results showed that the OTX2 promoter was activated in PK15 and PS23 cells, but not in PEF cells, indicating that the cloned OTX2 promoter retains cell-type specificity (Figure 6b). NANOG overexpression in piPSCs significantly reduced OTX2 expression (Figure $6 c$ ). The dose- and timedependent assays showed that the activity of the porcine OTX2 promoter could be repressed by NANOG in a dose-dependent manner (Figure 6d). To further monitor the NANOG binding region, the truncated OTX2 reporter constructs were made and used for luciferase assays (Figure $6 \mathrm{e}$ and Supplementary Figure S3). Within the $2 \mathrm{~kb}$ promoter region, there are multiple predicted binding sites of NANOG, OCT4, and SOX2. After removing NANOG binding sites in distal region of OTX2 promoter, OTX2 activity was not repressed by NANOG (Figure $6 f$ ). These observations indicate that NANOG can negatively regulate activity of porcine OTX2 promoter. Thus, the binding of NANOG to the OTX2 promoter could form negative feedback regulatory circuitry to regulate the self-renewal of porcine iPS cells.

\section{DISCUSSION}

As a homeodomain-containing TF in mammals, OTX2 regulation is essential for the normal development of brain and the genesis of photoreceptors. ${ }^{9-11,33}$ Also, Otx2 is described as the marker of the anterior central nervous system. ${ }^{34,35}$ The known Otx2 in several mammals is highly conserved and shares a conserved homeodomain region. High-level expression of Otx2 was detected in mouse/human brain. ${ }^{11,36,37}$ However, in pig, OTX2 is highly expressed not only in brain but also in reproductive tissues, as observed in this study. We found that porcine OTX2 transcripts initially existed in maternal components in oocytes, but were absent at two-cell and four-cell stages; the OTX2 level increased significantly after the eight-cell stage. In both mouse and human, ${ }^{25,26}$ Otx2 expression was upregulated along with embryonic development, indicating that OTX2 is an intrinsic TF activated 


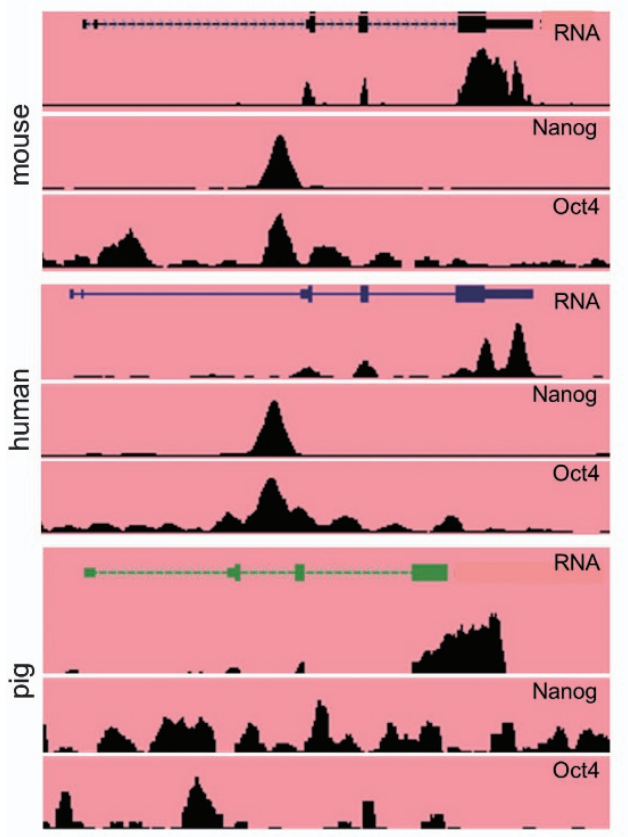

e

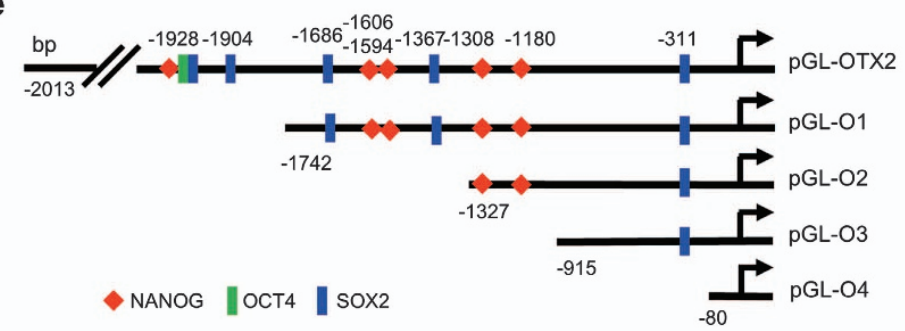

b

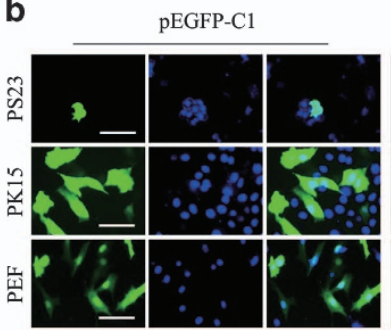

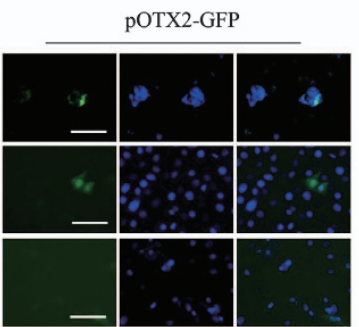

d

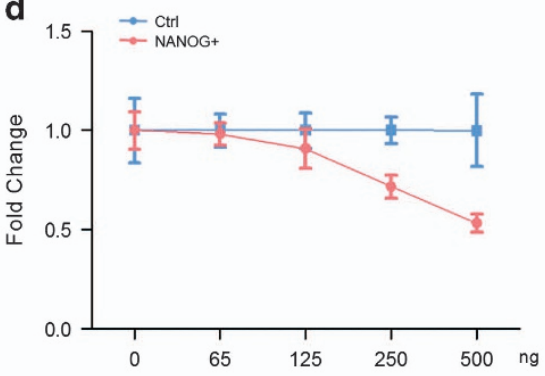

C

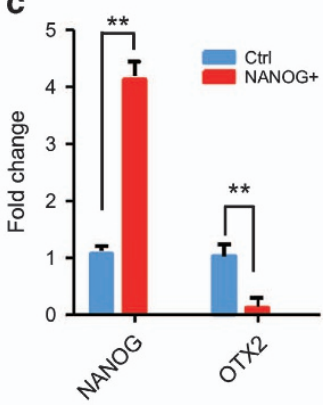

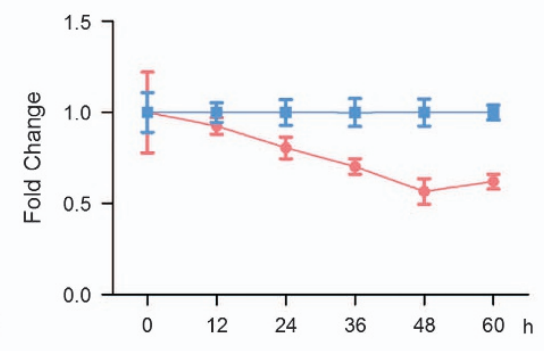

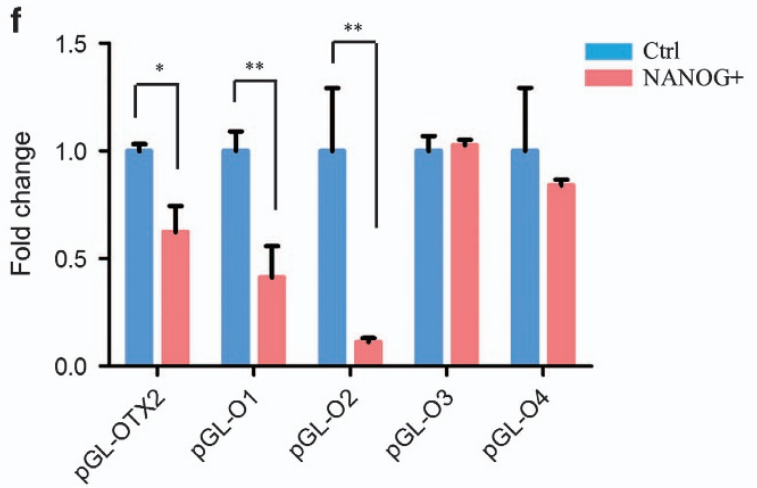

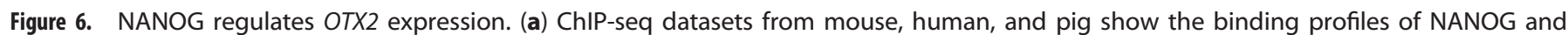
OCT4 associated with the OTX2 promoter loci. (b) GFP fluorescence assay. After 36 h transfection, OTX2-GFP-positive cells were present in PS23 and PK15 cells, but absent in PEF cells. Scale bar, $50 \mu \mathrm{m}$. (c) qRT-PCR analysis of NANOG and OTX2 expressions in PS23 cells cotransfected with pcDNA-NANOG. (d) pcDNA-NANOG and pGL-OTX2 were cotransfected into HEK-293 T cells for $36 \mathrm{~h}$. Dose-dependent assay (left) was performed with various concentrations of pcDNA-NANOG. Time-dependent assay (right) was performed with 250 ng of pcDNA-NANOG for various time points. (e) Diagram of reporter constructs with truncated OTX2 promoter. The predicted binding sites of NANOG, OCT4, and SOX2 are denoted. (f) Luciferase assay of OTX2 promoter activity in HEK-293 T cells that were cotransfected with pcDNA-NANOG and a truncated OTX2 vector. Ctrl, cells were transfected only with reporter pGL-OTX2. ${ }^{*} P<0.05,{ }^{* *} P<0.01, n=3$.

in the stage of zygotic genome activation and determines cell fate in early embryonic development. ${ }^{33}$ We also found that in porcine iPS cell lines, OTX2 expression was fourfold higher in primed piPSCs than in naive-like piPSCs (Figure 1g). Furthermore, the OTX2 expression level in metastable state cells was between the levels in naive-like and primed cells. These results indicate that OTX2 can be used as a marker to evaluate the pluripotent state of piPSCs. However, more precise determinations of cell type and culture condition must be applied to verify this hypothesis in future work.

We found that OTX2 and OCT4/SOX2 did not have the synergistic effect on promoting NANOG expression. Alternatively, overexpression of OTX2 alone could repress the expression of pluripotent genes in piPSCs. OTX2 has been shown to bind to the human tenascin-C promoter and transrepress tenascin activity. ${ }^{38,39}$ Investigations of regulatory circuitries of mouse Otx2 and other pluripotent factors have shown that Otx2 can interact with Oct4 and the two proteins cobind to primed-dominant enhancers to activate genes that promote exit from naive pluripotency. ${ }^{14,40}$ As a cell-state-specific regulator, Otx2 dynamically binds to enhancers, H3K4me1 and H3K27ac, that have low histone signals, and opens the previous inaccessible chromatin sites in which Oct4 and other TFs can cobind to these sites and promote enhancer-promoter interactions to elevate the expression of the set of genes that are related to lineage differentiation in primed cells. ${ }^{13}$ Thus, OTX2 is a negative regulator to influence iPS cell pluripotency. We assume that switching off porcine OTX2 expression during cellular reprogramming may help to gain the naive state piPSCs.

Of note, an abundance or lack of OTX2 in porcine iPS cells repressed and activated the NANOG promoter, respectively, in a time- and dose-dependent manner (Figure 5). Studies of singlecell expression profiling under different chemical and genetic perturbations showed that the expression of polycomb target genes, including Otx2, existed in a repressed yet poised state with a unique chromatin signature in iPSCs and negatively correlated with Nanog expression, representing regulators governing initial steps in lineage commitment. ${ }^{41}$ Similar observations were reported in mouse, in which constitutive and ubiquitous expression of Otx2 leads to a substantial reduction in Nanog expression; conversely, reduction of Otx2 causes strong activation of Nanog. ${ }^{12}$ In mouse Otx $2^{-1-}$ ESCs, cell colonies exhibited a sphere-like morphology, uniform AP staining, ubiquitous distribution of 
Nanog and Klf4, and a higher abundance Rex $1 .{ }^{12}$ In piPSCs, We found that knockdown of OTX2 with RNA-mediated interference also improved cell morphology and increased the expression of NANOG, OCT4, and ESRRB; however, KLF4 expression was unchanged and was independent of OTX2 activation, suggesting that other KLF family members may functionally overlap with KLF4, as reported previously, ${ }^{24}$ to regulate porcine cell reprogramming.

The ChIP-seq result in the pig sample displayed severe noise due to the low quality of the anti-NANOG antibody. ${ }^{32}$ ChIP-seq data from mouse and human showed that NANOG could directly bind to the Otx2 promoter region, which were also associated with Oct4 binding. A dose-dependent luciferase assay confirmed that NANOG repressed OTX2 activation (Figure 6). Our findings suggest that OTX2 and NANOG may form negative feedback regulatory circuitry to maintain pluripotent states in piPSCs.

We found that, unlike OCT4 and NANOG expression, SOX2 expression was positively correlated with OTX2 expression. Overexpression of OTX2 slightly elevated SOX2 expression, however, knockdown of OTX2 expression significantly reduced SOX2 expression (Figure 4), suggesting that the SOX2 protein, which also functions to specify neural lineage, is a determinant of cellular reprogramming potential and is required for epiblast maintenance. ${ }^{42,43}$ A previous study of Otx 2 and Sox 2 coregulation of Rax gene expression in fog showed that Otx2 overexpression was accompanied by increased expression of Sox 2 , in which the Otx2 protein directly bound to multiple cis-regulatory elements (N-2, AGATTA; N-3, GGATTA) that spatiotemporally control Sox2 expression. ${ }^{44,45}$ Thus, the regulatory function of the SOX2 protein in piPSCs may be different from that in mouse pluripotent stem cells. It would be interesting to investigate the transactivation role of SOX2 during the transition of pluripotent states in piPSCs.

In summary, we have identified OTX2 as an important cell-statespecific regulator of the fate and pluripotency of piPSCs, through inhibition of pluripotent gene expressions. We also showed that OTX2 and NANOG exhibited negative feedback circuitry to balance the pluripotency of piPSCs. Further studies will determine the functional relationship between OTX2 and other pluripotent factors and dissect the molecular mechanisms that maintain pluripotency in piPSCs.

\section{MATERIALS AND METHODS}

\section{Molecular cloning of OTX2 and vector construction}

Total RNAs were extracted from pig brain tissue by TRlzol Reagent (\#15596026, Invitrogen, Carlsbad, CA, USA) on the basis of the manufacturer's procedure. Pig OTX2 coding DNA sequence (CDS) and 3' UTR sequence were amplified by RT-PCR. The PCR fragments were cloned into the pMD18-T vector (RR420A, Takara, Dalian, China) and confirmed by DNA sequencing. The porcine OTX2 CDS was submitted to GenBank (Accession No. KP779653). To construct an OTX2 overexpression vector, the OTX2 CDS was subcloned into BamHI/Sall sites of pEGFP-C1 (\#6084-1, Clontech, Palo Alto, CA, USA) and BamHI/EcoRI sites of pcDNA3.1/N5-His (V385-20, Invitrogen) to generate the recombinant expression vector pEGFP-OTX2 that produces the GFP-OTX2 fusion protein and the recombinant expression vector pcDNA-OTX2 that produces OTX2 protein. To construct the pcDNA-NANOG plasmid, porcine NANOG CDS was subcloned into Hindlll/Xbal sites of pCDNA3.1/V5-His. OCT4 and SOX2 CDSs were subcloned into pMXs vector to generate pMX-OCT4 and pMX-SOX2.

To construct the OTX2 reporter vector, a $2 \mathrm{~kb}$ OTX2 promoter fragment was amplified by PCR and cloned into PGEM-T Easy vector (A1360, Promega, Madison, WI, USA) and confirmed by DNA sequencing. The porcine OTX2 promoter sequence was showed in Supplementary Figure S2 and also submitted to GenBank (Accession No. KR135411). The OTX2 promoter fragment was then subcloned into Xhol/Hindlll sites of pEGFP-1 (\#6086-1, Clontech) and pGL3-basic (U47295, Promega), respectively. The recombinant vectors were named as pOTX2-GFP and pGL-OTX2. To dissect the core regulatory region of the OTX2 promoter, we amplified four DNA fragments on the basis of $2 \mathrm{~kb}$ OTX2 promoter sequence and constructed four reporter vectors that retain the truncated OTX2 promoter.
The constructs were confirmed by restricted enzyme digestions (Supplementary Figure S3). The NANOG reporter vector pGL-NANOG and a series of deletion constructs, including pGL-N1, pGL-N2, and pGL-N3, were constructed as described previously. ${ }^{31}$

\section{Cell culture}

The porcine iPS cell line PS23 generated in this laboratory was cultured in piPS medium, ${ }^{22}$ which included knock-out DMEM (KO-DMEM, \#10829, Invitrogen) supplemented with 20\% FBS (\#16000-044, Gibco, Grand Island, NY, USA), $0.1 \mathrm{mM}$ nonessential amino acids (NEAA, \#11140-050, Invitrogen), $1 \mathrm{mM}$ L-glutamine (\#32571-093, Gibco), $10 \mathrm{ng} / \mathrm{ml}$ LIF (ESG1106, Millipore, Temecula, CA, USA), $10 \mathrm{ng} / \mathrm{ml}$ bFGF (GF003, Millipore), $0.1 \mathrm{mM}$ $\beta$-mercaptoethanol, 50 units $/ \mathrm{ml}$ penicillin/streptomycin, at $37^{\circ} \mathrm{C}$, in a $5 \%$ $\mathrm{CO}_{2}$ humidified atmosphere. PS23 cells were maintained on mitotically inactive mouse embryonic fibroblasts feeder derived from ICR mice, and passaged using $1 \mathrm{mg} / \mathrm{ml}$ collagenase type IV (\#17104-019, Gibco) and $0.05 \%$ trypsin (\#27250-018, Gibco) every 2 to 3 days. The mouse embryonic carcinoma cell line P19 was grown in alpha-MEM (\#11900-16, Gibco) with 10\% FBS (\#16000-044, Gibco). HEK-293 T cells were cultured in DMEM with $10 \%$ FBS at $37^{\circ} \mathrm{C}$, in a $5 \% \mathrm{CO}_{2}$ humidified atmosphere. Media were changed every 2 to 3 days.

\section{Cell transfection}

To evaluate OTX2 expression, cells were seeded in culture dishes $24 \mathrm{~h}$ before transfection. After reaching $80 \%$ confluence, $4.0 \mu \mathrm{g}$ pEGFP-OTX2 was transiently transfected into HEK-293 T cells plated on six-well plate using TurboFect transfection reagents (R0532, Thermo, Fair Lawn, NJ, USA) according to the manufacturer's instruction. After $36 \mathrm{~h}$, GFP-positive cells were examined and collected for western blotting. To determine the tissue specificity of the OTX2 promoter, the vector pOTX2-GFP was transfected into PEF, PK15, and PS23 cells, respectively, with Lipofectamine 2000 Regent (\#11668-019, Invitrogen) for $36 \mathrm{~h}$.

To overexpress OTX2 in porcine pluripotent stem cells, PS23 cells plated on a six-well plate were transiently transfected with $3.5 \mu \mathrm{g}$ pCDNA-OTX2 and pEGFP-OTX2, respectively, using Lipofectamine 2000 Regent for $36 \mathrm{~h}$. The transfection efficiency of pEGFP-OTX2 was over 35\%. To knockdown OTX2 expression in piPSCs, three small interfering RNAs (siRNAs; siR-543, siR-863, and siR-1115) and a negative control (siR-Ctrl) were synthesized (GenePharma, Shanghai, China), and the sequence of siRNAs are listed in Table 1. The 200 nM siRNA siR-543, siR-863, siR-1115, and siR-Ctrl were transiently transfected into PS23 cells, respectively, with Lipofectamine 2000 Regent for $36 \mathrm{~h}$. The transfection efficiency of siRNA determined with a fluorescent-labeled control siRNA (GenePharma) was more than $60 \%$. To increase siRNA interference efficiency, a second transfection of siRNA was conducted at $12 \mathrm{~h}$ after the first transfection, and samples were analyzed at $24 \mathrm{~h}$ after the second transfection.

To investigate NANOG interaction with the OTX2 promoter, pGL-OTX2, pcDNA-NANOG, and an internal control pRT-TK $(0.01 \mu \mathrm{g})$ were transiently cotransfected into HEK-293 T cells in a 48-well plate using TurboFect transfection reagents (R0532, Thermo). To determine the OTX2 regulatory effect on the NANOG promoter, deletion constructs, including pGL-NANOG, pGL-N1, pGL-N2, and pGL-N3, were transiently cotransfected with pcDNAOTX2 and pRT-TK into HEK-293 T cells. To explore interactions of OTX2, SOX2, and OCT4 with the NANOG promoter, vectors PCDNA-OTX2, pMXOCT4, and pMX-SOX2 with pGL-NANOG were transiently cotransfected into HEK-293 T cells for $36 \mathrm{~h}$.

\begin{tabular}{|lc|}
\hline Table 1. & siRNAs used in this study \\
\hline siRNA & \multicolumn{1}{c|}{ Sequence } \\
\hline siR-543 & F: 5'-GGGUUCAGGUAUGGUUUAATT-3' \\
& R: 5'-UUAAACCAUACCUGACCCTT-3' \\
siR-863 & F: 5'-GGAUAUGCUGGCUCAACUUTT-3' \\
& R: 5'-AAGUUGAGCCAGCAUAUCCTT-3' \\
siR-1115 & F: 5'-GCUGACUGCUUGGAUUAUTT-3' \\
& R: 5'-UAUAAUCCAAGCAGUCAGCTT-3' \\
siR-Ctrl & F: 5'-UUCUCCGACGUGUCACGUTT-3' \\
& R: 5'-ACGUGACACGUUCGGAGATT-3' \\
\hline
\end{tabular}


Luciferase assay

After $36 \mathrm{~h}$ transfection, cells were collected and lysed for $10 \mathrm{~min}$ at room temperature using passive lysis buffer (E194A, Promega). Luciferase activity was detected by luciferase assay reagents (E1960, Promega) and a BHP9504 microplate luminometer (D04407H, Hamamatsu, Beijing, China). Each treatment was measured in triplicate, and the average values of the ratio of firefly luciferase units to renilla luciferase units were used for data analysis. Statistical significance was accepted at $P<0.05$ and determined using the two-tailed $t$-test with equal variance.

\section{RT-PCR}

Total RNA from porcine tissues, pig fibroblasts, piPSCs, and embryos was extracted using TRIzol Reagent (\#15596-026, Invitrogen) according to the manufacturer's protocol. RNA samples were examined by measuring OD260/280 ratio of the optical density. RNAs with an optical density ratio of 2.0 were used for reverse transcription. One microgram of RNA was reverse-transcribed using RevertAid Reverse Transcriptase (EP0732, Thermo). The PCR was performed for 35 cycles at $94{ }^{\circ} \mathrm{C} 30 \mathrm{~s}, 56{ }^{\circ} \mathrm{C} 30 \mathrm{~s}$, and $72{ }^{\circ} \mathrm{C} 45 \mathrm{~s}$. PCR products were analyzed on $1.0 \%$ agarose gel. GAPDH was used as an internal control. To obtain the OTX2 $3^{\prime}$ UTR sequence, $1 \mu \mathrm{g}$ of RNA was reverse-transcribed using RevertAid Reverse Transcriptase with a $3^{\prime}$ UTR adapter primer. The first PCR reaction was performed for 20 cycles at $94^{\circ} \mathrm{C}$ $30 \mathrm{~s}, 60^{\circ} \mathrm{C} 30 \mathrm{~s}$, and $72{ }^{\circ} \mathrm{C} 1 \mathrm{~min}$ using the forward GSP1 primer. One microliter of PCR product was then utilized for the nested PCR, which was performed for 30 cycles at $94^{\circ} \mathrm{C} 30 \mathrm{~s}, 60^{\circ} \mathrm{C} 30 \mathrm{~s}$, and $72{ }^{\circ} \mathrm{C} 1 \mathrm{~min}$, with the forward GSP2 primer. GRT-PCR was performed in triplicate using SYBR Green PCR Master Mix (DRR420, Takara), and products were detected with the CFX96 real-time PCR system (Bio-Rad, Hercules, CA, USA). The reaction condition was: $95^{\circ} \mathrm{C}, 30 \mathrm{~s}$ as the first cycle, and 40 cycles of $95^{\circ} \mathrm{C} 5 \mathrm{~s}$ and $60^{\circ} \mathrm{C} 30 \mathrm{~s}$. Relative expression levels of genes were normalized to that of $G A P D H$ and calculated using $2^{-\triangle \Delta C t}$. To perform genomic DNA PCR, $1 \mu \mathrm{g}$ of genomic DNA was extracted from porcine brain tissue using a TIANamp Genomic DNA Kit (DP304-02, Tiangen Biotech, Beijing, China) as the template. PCR reactions were performed for 35 cycles at $94^{\circ} \mathrm{C} 30 \mathrm{~s}, 60^{\circ} \mathrm{C} 30 \mathrm{~s}$, and $72{ }^{\circ} \mathrm{C}$ $1 \mathrm{~min}$. PCR products were separated by $1.5 \%$ agarose gel electrophoresis. Primers used in this study are listed in Supplementary Table S1.

\section{Western blotting}

To determine the EGFP-OTX2 fusion protein, HEK-293 T cells were transfected with pEGFP-OTX2 and the control vector pEGFP-C1, respectively, using TurboFect transfection reagents (R0532, Thermo) for $36 \mathrm{~h}$. To determine the overexpressed or endogenous OTX2 protein, PS23 cells were transfected with pcDNA-OTX2 and siRNAs, respectively, for $36 \mathrm{~h}$. Cells were then collected by centrifugation. The $1 \times 10^{6}$ cell pellet was resuspended in SDS-PAGE loading buffer $(50 \mathrm{mM}$ Tris- $\mathrm{HCl} \mathrm{pH} 6.8,2 \%$ SDS, $10 \%$ glycerin, $2 \% \beta$-mercaptoethanol, and $0.05 \%$ bromophenol blue), and heated at $100{ }^{\circ} \mathrm{C}$ for $5 \mathrm{~min}$. A $20 \mu \mathrm{l}$ protein sample was subjected to $12 \%$ SDS-PAGE. After electrophoresis, proteins were transferred to a PVDF membrane (LC2002, Invitrogen) by semidry electrophoretic transfer (Bio-Rad) for $45 \mathrm{~min}$ at $15 \mathrm{~V}$. The membrane was blocked with blocking buffer $(20 \mathrm{mM}$ Tris/ $\mathrm{HCl} \mathrm{pH} \mathrm{7.6,137} \mathrm{mM} \mathrm{NaCl}, 0.1 \%$ Tween 20, and $8 \%$ dried skim milk) at $4{ }^{\circ} \mathrm{C}$ overnight, and then incubated with the primary anti-GFP antibody (1:5000, KM8009, Sungene Biotech, Tianjin, China), anti-GAPDH antibody (1:3000, KM9002, Sungene Biotech) and anti-OTX2 antibody $\left(1: 500\right.$, bs- $11958 \mathrm{R}$, Sungene Bioss, Beijing, China), respectively, at $37^{\circ} \mathrm{C}$ for $2 \mathrm{~h}$. After washing three times with TBS-T buffer ( $20 \mathrm{mM}$ Tris/HCl pH 7.6, $137 \mathrm{mM} \mathrm{NaCl}, 0.1 \%$ Tween 20), the membrane was incubated with a HRPconjugated secondary antibody $(1: 3000$, A0258, Beyotime, Shanghai, China) at $37^{\circ} \mathrm{C}$ for $1 \mathrm{~h}$. After washing in TBS-T for 10 min three times at room temperature, the membrane was incubated in enhanced chemiluminescent substrate (\#32106, Pierce, Rockford, IL, USA) for $1 \mathrm{~min}$ and detected with a Chemiluminescent ImagingSystem (ZY058176, Tanon4200, Shanghai, China).

\section{Alkaline phosphatase staining}

To perform AP staining, PS23 cells were washed twice using ice-cold phosphate buffered saline (PBS, pH 7.4), fixed with $4 \%$ paraformaldehyde in PBS for $10 \mathrm{~min}$ at room temperature, and washed three times using icecold PBS. Cells were then incubated at room temperature in $0.1 \mathrm{M}$ Tris buffer, pH 7.4, with $1.0 \mathrm{mg} / \mathrm{ml}$ Fast Red TR, $0.4 \mathrm{mg} / \mathrm{ml}$ Naphthol AS-MX Phosphate (\#1596-56-1, Sigma, St Louis, MO, USA). After 10 min incubation, AP-positive iPS colonies were identified by their red color.
Data sources and bioinformatics analysis

Protein sequences used in phylogenetic analysis were obtained from the GenBank database. GenBank database for OTX protein is under the following accession numbers: pig, XP_005660050.1; mouse, NP_001273412.1; human, NP_001257453.1; rat, NP_001094036.1; dog, XP_547830.2; cat, XP_006932953.1; horse, XP_005605262.1; cattle, NP_001180130.1; rabbit, XP_008267928.1; shrew, XP_004584906.1; baboon, XP_009209859.1; marmoset, JAB21705.1; and hedgehog, NP_571326.1. ChIP-seq data were from the ChIP-seq dataset (http://www.cepbrowser.org/). ChIP-seq data were obtained from the ChIP-seq dataset. ${ }^{32}$ Pig embryo RNA-seq data were obtained from the Lab Archive (http://www.ncbi.nlm.nih.gov/sra) under accession number SRA076823. ${ }^{30}$ RNA-seq data for piPS-w cells, ${ }^{18}$ piPS-j cells, ${ }^{23}$ and piPS-g cells ${ }^{24}$ were obtained from the EMBL-EBI database (http://www.ebi.ac.uk/) under accession number E-MTAB-2634. Phylogenetic analysis of OTX2 was performed using online software (http://phylogeny.lirmm.fr/phylo_cgi/index. cgi). ${ }^{46,47}$ Genome analysis was performed with the UCSC Genome Browser website (http://genome.ucsc.edu) ${ }^{48}$ and the Ensembl Genome Browser (http:// www.ensembl.org/index.html). Multiple sequence alignments were performed by Clustal Omega (http://www.ebi.ac.uk/Tools/msa/clustalo/). Putative sites of TFs were predicted by JASPAR software (http://jaspar.cgb.ki.se/). ${ }^{49,50}$ Conserved domain analysis was performed by the Conserved Domain Database (http://www.ncbi.nlm.nih.gov/Structure/cdd/cdd.shtml). ${ }^{27,28}$

\section{Statistical analysis}

Data are presented as mean \pm S.D. The S.D. in this study was calculated in three replicates. Statistical significance was assessed using the Student's $t$-test where indicated in the figure legends.

\section{ACKNOWLEDGEMENTS}

This work was supported by the National Natural Science Foundation of China (No. 31571521 and 31371505).

\section{COMPETING INTEREST}

The authors declare no conflict of interest.

\section{REFERENCES}

1 Nichols J, Smith A. Naive and primed pluripotent states. Cell Stem Cell 2009; 4: 487-492.

2 Hanna JH, Saha K, Jaenisch R. Pluripotency and cellular reprogramming: facts, hypotheses, unresolved issues. Cell 2010; 143: 508-525.

3 Niwa H. How is pluripotency determined and maintained? Development 2007; 134: 635-646.

4 Niwa H, Ogawa K, Shimosato D, Adachi K. A parallel circuit of LIF signalling pathways maintains pluripotency of mouse ES cells. Nature 2009; 460: 118-122.

5 Ying QL, Wray J, Nichols J, Batlle-Morera L, Doble B, Woodgett J et al. The ground state of embryonic stem cell self-renewal. Nature 2008; 453: 519-523.

6 Lanner F, Rossant J. The role of FGF/Erk signaling in pluripotent cells. Development 2010; 137: 3351-3360.

7 Najm FJ, Chenoweth JG, Anderson PD, Nadeau JH, Redline RW, McKay RD et al. Isolation of epiblast stem cells from preimplantation mouse embryos. Cell Stem Cell 2011; 8: 318-325.

8 Young RA. Control of the embryonic stem cell state. Cell 2011; 144: 940-954.

9 Boyl PP, Signore M, Acampora D, Martinez-Barbera JP, llengo C, Annino A et al. Forebrain and midbrain development requires epiblast-restricted Otx2 translational control mediated by its 3' UTR. Development 2001; 128: 2989-3000.

10 Vernay B, Koch M, Vaccarino F, Briscoe J, Simeone A, Kageyama R et al. Otx2 regulates subtype specification and neurogenesis in the midbrain. $J$ Neurosci 2005; 25: 4856-4867.

11 Beby F, Lamonerie T. The homeobox gene Otx2 in development and disease. Exp Eye Res 2013; 111: 9-16.

12 Acampora D, Di Giovannantonio LG, Simeone A. Otx2 is an intrinsic determinant of the embryonic stem cell state and is required for transition to a stable epiblast stem cell condition. Development 2013; 140: 43-55.

13 Buecker C, Srinivasan R, Wu ZX, Calo E, Acampora D, Faial T et al. Reorganization of enhancer patterns in transition from naive to primed pluripotency. Cell Stem Cell 2014; 14: 838-854.

14 Yang SH, Kalkan T, Morissroe C, Marks H, Stunnenberg H, Smith A et al. Otx2 and Oct4 drive early enhancer activation during embryonic stem cell transition from naive pluripotency. Cell Rep 2014; 7: 1968-1981. 
15 Festuccia N, Osorno R, Halbritter F, Karwacki-Neisius V, Navarro P, Colby D et al. Esrrb is a direct Nanog target gene that can substitute for Nanog function in pluripotent cells. Cell Stem Cell 2012; 11: 477-490.

16 Saunders A, Faiola F, Wang J. Concise review: pursuing self-renewal and pluripotency with the stem cell factor Nanog. Stem Cells 2013; 31: 1227-1236.

17 Chambers I, Colby D, Robertson M, Nichols J, Lee S, Tweedie S et al. Functional expression cloning of Nanog, a pluripotency sustaining factor in embryonic stem cells. Cell 2003; 113: 643-655.

18 West FD, Terlouw SL, Kwon DJ, Mumaw JL, Dhara SK, Hasneen K et al. Porcine induced pluripotent stem cells produce chimeric offspring. Stem Cells Dev 2010; 19: $1211-1220$

19 Wu Z, Chen J, Ren J, Bao L, Liao J, Cui C et al. Generation of pig induced pluripotent stem cells with a drug-inducible system. J Mol Cell Biol 2009; 1: 46-54.

20 Esteban MA, Xu J, Yang J, Peng M, Qin D, Li W et al. Generation of induced pluripotent stem cell lines from Tibetan miniature pig. J Biol Chem 2009; 284: 17634-17640.

21 Ezashi T, Telugu BP, Alexenko AP, Sachdev S, Sinha S, Roberts RM. Derivation of induced pluripotent stem cells from pig somatic cells. Proc Natl Acad Sci USA 2009; 106: 10993-10998.

22 Cheng D, Guo Y, Li Z, Liu Y, Gao X, Gao Y et al. Porcine induced pluripotent stem cells require LIF and maintain their developmental potential in early stage of embryos. PloS One 2012; 7: e51778.

23 Fujishiro SH, Nakano K, Mizukami Y, Azami T, Arai Y, Matsunari H et al. Generation of naive-like porcine-induced pluripotent stem cells capable of contributing to embryonic and fetal development. Stem Cells Dev 2013; 22: 473-482.

24 Zhang S, Guo Y, Cui Y, Liu Y, Yu T, Wang H. Generation of intermediate porcine iPS cells under culture condition favorable for mesenchymal-to-epithelial transition. Stem Cell Rev 2015; 11: 24-38.

25 Larsen KB, Lutterodt M, Rath MF, Moller M. Expression of the homeobox genes PAX6, OTX2, and OTX1 in the early human fetal retina. Int J Dev Neurosci 2009; 27: $485-492$

26 Simeone A, Acampora D, Mallamaci A, Stornaiuolo A, D'Apice MR, Nigro V et al. A vertebrate gene related to orthodenticle contains a homeodomain of the bicoid class and demarcates anterior neuroectoderm in the gastrulating mouse embryo. Embo J 1993; 12: 2735-2747.

27 Marchler-Bauer A, Zheng C, Chitsaz F, Derbyshire MK, Geer LY, Geer RC et al. CDD: conserved domains and protein three-dimensional structure. Nucleic Acids Res 2013; 41: D348-D352.

28 Marchler-Bauer A, Derbyshire MK, Gonzales NR, Lu S, Chitsaz F, Geer LY et al. CDD: NCBI's conserved domain database. Nucleic Acids Res 2015; 43: D222-D226.

29 Kaur R, Aiken C, Morrison LC, Rao R, Del Bigio MR, Rampalli $S$ et al. OTX2 exhibits cell-context-dependent effects on cellular and molecular properties of human embryonic neural precursors and medulloblastoma cells. Dis Model Mech 2015; 8: 1295-1309.

30 Cao S, Han J, Wu J, Li Q, Liu S, Zhang W et al. Specific gene-regulation networks during the pre-implantation development of the pig embryo as revealed by deep sequencing. BMC Genomics 2014; 15: 4.

31 Yang F, Zhang J, Liu Y, Cheng D, Wang H. Structure and functional evaluation of porcine NANOG that is a single-exon gene and has two pseudogenes. Int J Biochem Cell Biol 2015; 59: 142-152.

32 Xiao S, Xie D, Cao X, Yu P, Xing X, Chen CC et al. Comparative epigenomic annotation of regulatory DNA. Cell 2012; 149: 1381-1392.

33 Muranishi Y, Terada K, Inoue T, Katoh K, Tsujii T, Sanuki R et al. An essential role for RAX homeoprotein and NOTCH-HES signaling in Otx2 expression in embryonic retinal photoreceptor cell fate determination. J Neurosci 2011; 31: 16792-16807.

$34 \mathrm{Li} J Y$, Joyner AL. Otx2 and Gbx2 are required for refinement and not induction of mid-hindbrain gene expression. Development 2001; 128: 4979-4991.
35 Gallegos-Cardenas A, Webb R, Jordan E, West R, West FD, Yang JY et al. Pig induced pluripotent stem cell-derived neural rosettes developmentally mimic human pluripotent stem cell neural differentiation. Stem Cells Dev 2015; 24: 1901-1911.

36 Di Salvio M, Di Giovannantonio LG, Omodei D, Acampora D, Simeone A. Otx2 expression is restricted to dopaminergic neurons of the ventral tegmental area in the adult brain. Int J Dev Biol 2010; 54: 939-945.

37 Simeone A, Acampora D, Gulisano M, Stornaiuolo A, Boncinelli E. Nested expression domains of four homeobox genes in developing rostral brain. Nature 1992; 358: 687-690.

38 Gherzi R, Briata P, Boncinelli E, Ponassi M, Querze G, Viti F et al. The human homeodomain protein OTX2 binds to the human tenascin-C promoter and transrepresses its activity in transfected cells. DNA Cell Biol 1997; 16: 559-567.

39 Ghatnekar A, Trojanowska M. GATA-6 is a novel transcriptional repressor of the human Tenascin-C gene expression in fibroblasts. Biochim Biophys Acta 2008; 1779: 145-151.

40 Yang SH, Kalkan T, Morrisroe C, Smith A, Sharrocks AD. A genome-wide RNAi screen reveals MAP kinase phosphatases as key ERK pathway regulators during embryonic stem cell differentiation. PLoS Genetics 2012; 8: e1003112.

41 Kumar RM, Cahan P, Shalek AK, Satija R, DaleyKeyser AJ, Li H et al. Deconstructing transcriptional heterogeneity in pluripotent stem cells. Nature 2014; 516: 56-61.

42 Han DW, Tapia N, Arauzo-Bravo MJ, Lim KT, Kim KP, Ko K et al. Sox2 level is a determinant of cellular reprogramming potential. PloS One 2013; 8: e67594.

43 Avilion AA, Nicolis SK, Pevny LH, Perez L, Vivian N, Lovell-Badge R. Multipotent cell lineages in early mouse development depend on SOX2 function. Genes Dev 2003; 17: 126-140.

44 Uchikawa M, Ishida Y, Takemoto T, Kamachi Y, Kondoh H. Functional analysis of chicken Sox2 enhancers highlights an array of diverse regulatory elements that are conserved in mammals. Dev Cell 2003; 4: 509-519.

45 Danno H, Michiue T, Hitachi K, Yukita A, Ishiura S, Asashima M. Molecular links among the causative genes for ocular malformation: Otx2 and Sox2 coregulate Rax expression. Proc Natl Acad Sci USA 2008; 105: 5408-5413.

46 Dereeper A, Audic S, Claverie JM, Blanc G. BLAST-EXPLORER helps you building datasets for phylogenetic analysis. BMC Evol Biol 2010; 10: 8.

47 Dereeper A, Guignon V, Blanc G, Audic S, Buffet S, Chevenet F et al. Phylogeny.fr: robust phylogenetic analysis for the non-specialist. Nucleic Acids Res 2008; 36: W465-W469.

48 Karolchik D, Barber GP, Casper J, Clawson H, Cline MS, Diekhans M et al. The UCSC Genome Browser database: 2014 update. Nucleic Acids Res 2014; 42: D764-D770.

49 Mathelier A, Zhao X, Zhang AW, Parcy F, Worsley-Hunt R, Arenillas DJ et al. JASPAR 2014: an extensively expanded and updated open-access database of transcription factor binding profiles. Nucleic Acids Res 2014; 42: D142-D147.

50 Sandelin A, Alkema W, Engstrom P, Wasserman WW, Lenhard B et al. an openaccess database for eukaryotic transcription factor binding profiles. Nucleic Acids Res 2004; 32: D91-D94.

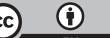

This work is licensed under a Creative Commons Attribution 4.0 International License. The images or other third party material in this article are included in the article's Creative Commons license, unless indicated otherwise in the credit line; if the material is not included under the Creative Commons license, users will need to obtain permission from the license holder to reproduce the material. To view a copy of this license, visit http://creativecommons.org/licenses/ by/4.0/

(c) The Author(s) 2016

Supplemental Information accompanies the paper on the Cell Death and Discovery website (http://www.nature.com/cddiscovery) 